\title{
MicroRNA-328 inhibits migration and epithelial- mesenchymal transition by targeting CD44 in nasopharyngeal carcinoma cells
}

This article was published in the following Dove Press journal:

OncoTargets and Therapy

\author{
Chien-Hung Lin ${ }^{1,2}$ \\ Ming-Chang Chiang ${ }^{3}$ \\ Yann-Jang Chen ${ }^{1,4,5}$ \\ 'Institute of Clinical Medicine, \\ National Yang-Ming University, Taipei, \\ Taiwan; ${ }^{2}$ Department of Pediatrics, \\ Zhongxing Branch, Taipei City \\ Hospital, Taipei, Taiwan; ${ }^{3}$ Department \\ of Life Science, College of Science \\ and Engineering, Fu Jen Catholic \\ University, New Taipei City, Taiwan; \\ ${ }^{4}$ Department of Life Sciences and \\ Institute of Genome Sciences, \\ National Yang-Ming University, Taipei, \\ Taiwan; ${ }^{5}$ Department of Pediatrics, \\ Renai Branch, Taipei City Hospital, \\ Taipei, Taiwan
}

Background: MicroRNAs (miRNAs) play crucial roles in various types of cancers, particularly in tumor development, migration, and progression. Dysregulation of miR-328 was reported to occur in some types of human malignancies, however, the role of miR-328 in nasopharyngeal carcinoma (NPC) and its potential involvement in metastasis remain undetermined.

Methods: The invasion capacity of NPC sphere-forming cells was evaluated by in vitro cell migration assays. Differential miRNAs expression was examined in NPC sphere-forming cells compared to parental monolayer cells using miRNA array analysis. The role of miR-328 in regulating NPC cells migratory properties was analyzed after miR-328 mimics transfection. The expression of E-cadherin and CD44 was analyzed by flow cytometry. CD44 was examined as a target of miR-328 through luciferase reporter assays and Western blotting.

Results: Here, we report that NPC TW01 and TW06 sphere-forming cells exhibited increased migratory ability in comparison with parental monolayer cells. Sphere-forming cells had significantly lower levels of miR-328, as observed using miRNA arrays and confirmed through real-time polymerase chain reaction. Overexpression of miR-328 induced by transfection with synthetic miR-328 mimics decreased the migration of NPC sphere-forming cells. The inhibitory effects were associated with increased expression of E-cadherin and the downregulated expression of mesenchymal markers such as N-cadherin, Snail, and vimentin. Moreover, our results demonstrated that miR-328 suppressed NPC cell migration and inhibited the epithelial-mesenchymal transition process directly through a binding site on the CD44 3' untranslated region.

Conclusion: miR-328, a previously unrecognized miRNA, may serve as a potential prognostic marker and therapeutic target for NPC.

Keywords: miR-328, EMT, CD44, NPC, cancer cell migration

\section{Introduction}

Nasopharyngeal carcinoma (NPC) is a malignant disease originating in the nasopharynx and is prevalent among southern Chinese and Taiwanese populations. Many studies have concluded that NPC is a complex disease attributable to interactions among a genetic predisposition, environmental factors, and Epstein-Barr virus infection. ${ }^{1}$ The challenges pertaining to improving the therapeutic efficacy and enhancing the survival rate of NPC patients have still not been overcome. Although NPC is radiation sensitive, the outcomes of patients with advanced stages of the disease are not satisfactory, owing to local recurrence and chemoresistance. ${ }^{2,3}$ The outcomes remain poor in a substantial number of NPC patients, with relapse and distant metastasis often being reported. ${ }^{4,5}$ Hence, it is imperative to study the molecular mechanisms underlying the progression of NPC to improve the prognosis. 
Accumulating evidence suggests that microRNAs (miRNAs) play pertinent roles in various types of cancers and are involved in tumor development and progression. ${ }^{6}$ Most miRNAs target mRNAs by binding to the $3^{\prime}$ untranslated region (UTR) and disturbing the expression of genes. miRNAs can serve as targets in direct therapy for tumors and as biomarkers to predict prognosis. ${ }^{7}$ In addition, the roles of dysregulated miRNA in the pathogenesis of NPC have been reported; specifically, miRNAs play crucial roles in NPC tumorigenesis and may serve as potential biomarkers and therapeutic targets. ${ }^{8-10}$ However, the expression of miRNAs during NPC recurrence and metastasis is still largely unexplored. Literature characterizing the functions and mechanisms of miRNAs in NPC metastasis remains limited. An in-depth understanding of the relationship between miRNAs and tumor relapse will help us to identify novel biomarkers and thus improve therapeutic efficacy and clinical outcomes.

Tumor cells often undergo an epithelial-mesenchymal transition (EMT) process during metastasis and are characterized by reduced intercellular adhesion and increased migratory properties. ${ }^{11}$ EMT-type tumor cells exhibit an invasive phenotype and are closely associated with tumor cell invasion and therapeutic difficulty. ${ }^{12}$ Notably, miRNAs were reported to regulate EMT in a number of studies. For example, the miR-200 family was shown to directly target Nanog to inhibit EMT, but may play an oncogenic role in NPC, ${ }^{13,14}$ whereas miR-30 was shown to target Snaill to inhibit invasion and metastasis in the regulation of EMT. ${ }^{15}$

CD44 is a widely expressed cell-surface transmembrane glycoprotein and is associated with cell-matrix and cell-cell interactions through hyaluronate and other extracellular components. ${ }^{16}$ Furthermore, it can regulate a variety of processes and play a valuable role in tumor cell adhesion, migration, and the capacity to undergo metastases related to EMT ${ }^{17}$ Previous studies have implicated CD44 as a marker of NPC tumors, which are characterized by a highly invasive and metastatic phenotype. . $^{18,19}$

In this study, we discovered that NPC cells growing nonadherently could express the EMT phenotype, and these sphere-forming cells had different miRNA expression profiles compared with parental monolayer cells. The NPC sphere-forming cells had considerably lower levels of miR-328 upon examination using miRNA arrays and confirmation through real-time polymerase chain reaction (PCR). Further investigation showed that overexpression of miR-328 could inhibit migration by reversing EMT in NPC cells. CD44 was validated as a target of miR-328 through
3'UTR luciferase assays and Western blot analysis. Our results indicated that increased expression of miR-328 may prevent the invasion and metastasis of NPC cells through suppression of CD44; thus, miR-328 is a potentially crucial marker and a therapeutic target for NPC.

\section{Materials and methods Cell culture}

Parental monolayer cell culture: NPC TW01 (WHO type I, keratinizing squamous cell carcinoma) and TW06 (WHO type III, undifferentiated carcinoma) cell lines ${ }^{20}$ were gifted from Chih-Hsin Ou-Yang (National Yang-Ming University, Taipei, Taiwan) and were cultured in $10 \mathrm{~cm}^{2}$ dishes with DMEM (Thermo Fisher Scientific, Waltham, MA, USA); $10 \%$ fetal bovine serum (Biological Industries, Kibbutz Beit Haemek, Israel); 1\% sodium pyruvate (Biological Industries); $1 \%$ penicillin, streptomycin, and amphotericin (Biological Industries); and 1\% nonessential amino acids (Biological Industries). The cells were incubated at $37^{\circ} \mathrm{C}$ in a humidified atmosphere of $5 \% \mathrm{CO}_{2}$. Nonadherent cell culture: NPC TW01 and TW06 cells were seeded nonadhesively at a density of $2 \times 10^{4} / \mathrm{mm}^{3}$ in six-well culture dishes coated with a thin agarose layer and containing serum-free DMEM/F12 medium (Thermo Fisher Scientific). The culture medium was changed every other day until sphere formation. After 7-10 days, the spheres were collected by filtration through a $70-\mu \mathrm{m}$ mesh and used in subsequent experiments.

\section{Migration assay}

In vitro cell migration assays were performed in BD Falcon cell culture inserts (BD Biosciences, San Jose, CA, USA). Cells $\left(1 \times 10^{5}\right)$ suspended in $100 \mu \mathrm{L}$ of serum-free DMEM were seeded in the upper compartment, while the lower compartments were filled with $1 \mathrm{~mL}$ of DMEM containing $10 \%$ fetal calf serum. After incubation for $24 \mathrm{~h}$ at $37^{\circ} \mathrm{C}$ with $5 \%$ $\mathrm{CO}_{2}$, the nonmigrating cells were scrubbed from the upper surface of the membrane. The migrating cells on the reverse side were stained with $0.1 \%$ crystal violet and counted under a light microscope.

\section{RNA extraction and real-time quantitative reverse transcription PCR (qRT-PCR)}

Total RNA was isolated with Trizol reagent (Thermo Fisher Scientific). First-strand cDNA was reverse transcribed according to the manufacturer's protocols. 
The primer sequences used for qRT-PCR were as follows:

Glyceraldehyde-3-phosphate dehydrogenase (GAPDH)

Forward: 5'-ACGGGAAGCTCACTGGCATGG-3'

Reverse: 5'-GGTCCACCACCCTGTTGCTGTA-3'

N-Cad Forward: 5'-AGGGTGGACGTCATTGTAGC-3'

Reverse: 5'-CTGTTGGGGTCTGTCAGGAT-3'

Vim Forward: 5'-GAGAACTTTGCCGTTGAAGC-3'

Reverse: 5'-GCTTCCTGTAGGTGGCAATC- $3^{\prime}$

Snail Forward: 5'-CTTCCAGCAGCCCTACGAC-3'

Reverse: 5'-CGGTGGGGTTGAGGATCT-3'

E-Cad Forward: 5'-TGCCCAGAAAATGAAAAAGG-3'

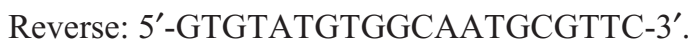

\section{miRNA array analysis}

RNA for miRNA expression profiling was reverse transcribed into cDNA by using Megaplex ${ }^{\mathrm{TM}}$ RT Primer Human Pool sets and preamplified by using Megaplex PreAmp Human Primer Pool sets (Applied Biosystems, Foster City, CA, USA). The resulting cDNA was loaded onto TaqMan Low Density miRNA Array (TLDA) cards, according to the manufacturer's instructions. The TLDA cards were run on an ABI 7900HT real-time PCR system.

\section{Small RNA extraction and quantitation of miR-328 through qRT-PCR}

Small RNA was extracted using the mirVana miRNA Isolation Kit (Applied Biosystems) and was reverse transcribed using the TaqMan microRNA Reverse Transcription Kit (Applied Biosystems). The sequence-specific forward primers for miR-328 and a U6 internal control were 5'-CUGGCCCUCUCUGCCCUUCCGU- ${ }^{\prime}$ and $5^{\prime}$-CTCGC TTCGGCAGCACATATA-3', respectively. Single TaqMan ${ }^{\circledR}$ microRNA assays (Applied Biosystems) that include RT primers and TaqMan probes were used to quantify the levels of hsa-miR-328 and the U6 internal control as per the manufacturer's instructions. All PCR reactions were run in triplicate.

\section{Transfection of miRNAs mimic}

An miR-328 mimic and negative control were obtained from Dharmacon (Lafayette, CO, USA). The miR-328 mimic with either control (Dharmacon) was diluted in $1 \mathrm{~mL}$ of growth medium to a final concentration of $10 \mathrm{nM}$. The solutions were mixed gently and incubated at room temperature for $15 \mathrm{~min}$. Transfection was performed using GenMute siRNA Transfection Reagent (SignaGen Laboratories, Rockville, MD, USA) according to the manufacturer's instructions. Six-well plates were seeded with $2 \times 10^{4}$ cells and incubated at $37^{\circ} \mathrm{C}$ with $5 \% \mathrm{CO}_{2}$ for $24 \mathrm{~h}$. Subsequently, cells were transfected with the miR-328 mimic and the negative control according to the manufacturer's protocol.

\section{Flow cytometry for antibody analysis}

Fluorescein isothiocyanate (FITC)-conjugated anti-E-cadherin (Abcam, Cambridge, MA, USA) was employed to assess the cell-surface antigen phenotype. The anti-CD44-FITC (BD Biosciences) antibody was used to detect CD44 on the cell surface. The cells were then incubated at $4^{\circ} \mathrm{C}$ for $15 \mathrm{~min}$ in the dark. Following incubation, they were washed with cold fluorescence activated cell sorting buffer. The labeled cells were analyzed using the Gallios flow cytometer (Beckman Coulter, Brea, CA, USA), and the data were interpreted with FlowJo software.

\section{Western blot analysis}

Cells were lysed in radioimmunoprecipitation assay (RIPA) buffer containing a $1 \times$ protease inhibitor cocktail, and protein concentrations were determined using the Bradford assay (Bio-Rad, Philadelphia, PA, USA). Total protein $(10 \mu \mathrm{g})$ was boiled at $95^{\circ} \mathrm{C}$ for $5 \mathrm{~min}$, separated through $12 \%$ sodium dodecyl sulfate-polyacrylamide gel electrophoresis, and transferred onto a polyvinylidene difluoride membrane. The membranes were blocked with $5 \%$ nonfat milk, incubated overnight at $4{ }^{\circ} \mathrm{C}$ with a primary antibody against CD44, and then incubated at room temperature with a secondary antibody. A rabbit anti-GAPDH antibody was used as a sample-loading control. After the membranes were washed four times for 15 min with $\mathrm{NaCl} /$ Tris/Tween-20 at room temperature, the immunoreactivity was visualized using an enhanced chemiluminescence detection system.

\section{Luciferase assay}

TW01 sphere-forming cells were cotransfected with reporter plasmids containing 3'UTR CD44 and the miR-328 mimicnegative control or miR-328 mimic. Cells were collected $48 \mathrm{~h}$ after transfection, and luciferase assays were performed using a Secrete-Pair ${ }^{\mathrm{TM}}$ Duo-Luciferase Assay Kit (GeneCopoeia, Rockville, MD, USA). Luciferase activity was normalized relative to the control activity.

\section{Statistical analysis}

Data were expressed as the mean \pm SD from a minimum of three separate experiments. The differences between two groups were analyzed using the Student's $t$-test. 
The differences among three groups were analyzed using one-way or two-way analysis of variance. All statistical analyses were performed with SPSS 17.0 software, and $P$-values $<0.05$ were defined as statistically significant.

\section{Ethical approval}

Prior approval was obtained from the Ethics Committee of Taipei City Hospital, which included the use of the gifted cell lines.

\section{Results}

\section{NPC sphere-forming cells exhibit mesenchymal phenotypes and promote cell migration}

Two human NPC cell lines, TW01 and TW06, were used in an adherent monolayer or nonadherent suspended culture condition. We observed that the NPC cells in the nonadherent culture could grow into spheres, were spindle shaped, and were less tightly bound than the parental cells in adherent monolayer culture (Figure 1A). The contrasting migratory properties of the parental monolayer and sphere-forming cells were demonstrated through a Transwell migration assay (Figure 1B and C). These results indicated a trend of increased migratory ability among the sphere-forming cells. We also determined the expression level of E-cadherin among these NPC cells and observed that its level in sphere-forming cells was significantly lower than that in parental monolayer cells (Figure 1D). These results revealed that NPC sphere-forming cells express the characteristics of EMT, and the mesenchymal phenotype was associated with the acquisition of invasive behavior.

\section{Determination of invasion-related miRNAs among NPC cells using miRNA \\ arrays}

To determine the specific miRNAs that correlate with the migratory state of NPC cells, miRNA array experiments were conducted, and the differential expression of miRNAs determined using the $\Delta \Delta \mathrm{CT}$ method was compared between the two groups (Figure S1). As shown in Figure 2A, the expression levels of miRNA-328 were significantly lower in the sphere-forming cells than in the parental monolayer cells. The expression levels were confirmed through real-time PCR (Figure 2B) and were consistent with those in miRNA array analysis. These data suggested that miR-328 is involved in the migration of TW01 and TW06 cells, and its dysregulated expression may play a key role in the metastatic ability of NPC cells.

\section{Migration was associated with the expression of miRNA-328}

To analyze the role of miR-328 in NPC cells, we overexpressed it by transfecting cells with a specific miR-328 mimic and assessed the migratory ability of NPC cells using a Transwell migration assay. The expression level of miR-328 was determined through real-time PCR. The data demonstrated that the mRNA expression of miR-328 in the mimic group was significantly higher than that in the control group $(P<0.01$; Figure $3 \mathrm{~A})$. Following transfection of the sphere-forming cells with the mimic, Transwell migration assays were performed to determine their migratory ability. Our results showed that the overexpression of miRNA-328 in TW01 and TW06 sphere-forming cells could suppress their ability to migrate. The percentage of migratory cells was significantly decreased in the sphere-forming cells overexpressing miRNA-328 (Figure 3B). These results demonstrated that the expression of miRNA-328 may affect the migration property of NPC cells and thereby lessen their aggressiveness.

\section{miRNA-328 mediates the EMT of NPC cells}

To study whether miR-328 affects the expression of EMT markers, we analyzed the mRNA levels of mesenchymal cell markers (N-cadherin, vimentin, and Snail) and an epithelial cell marker (E-cadherin) using qRT-PCR. We observed that the expression of E-cadherin in TW01 sphere-forming cells increased after transfection. Furthermore, transfection of TW01 sphere-forming cells with the mimic significantly reduced the expression of $\mathrm{N}$-cadherin, vimentin, and Snail in comparison with that in the control group (Figure 4A). Our results showed that the overexpression of miR-328 resulted in an increase in E-cadherin expression and significant reductions in N-cadherin, vimentin, and Snail expression with respect to the control cells. These results indicated that upregulated miR-328 expression could influence the expression levels of EMT markers and thereby inhibit the migratory ability of NPC cells. We then investigated whether the overexpression of miRNA-328 affects the expression of CD44 in TW01 and TW06 sphere-forming cells. Flow cytometric analysis revealed that the overexpression of miRNA-328 resulted in the decreased expression of CD44 in TW01 and TW06 sphere-forming cells (Figure 4B). These data suggested that miRNA-328 may suppress 


\section{A}

TW01 parental monolayer cells

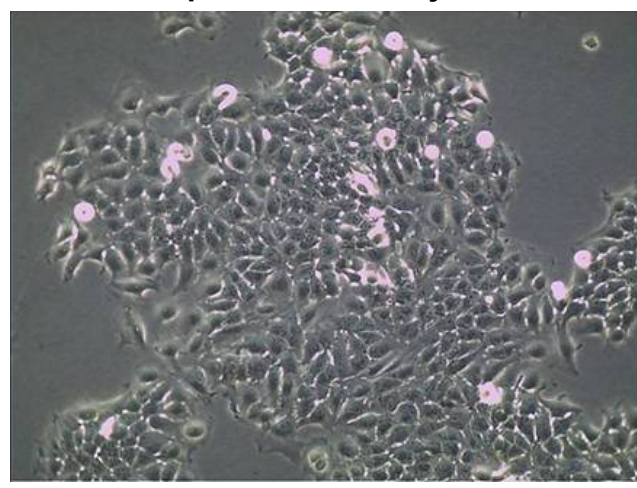

B

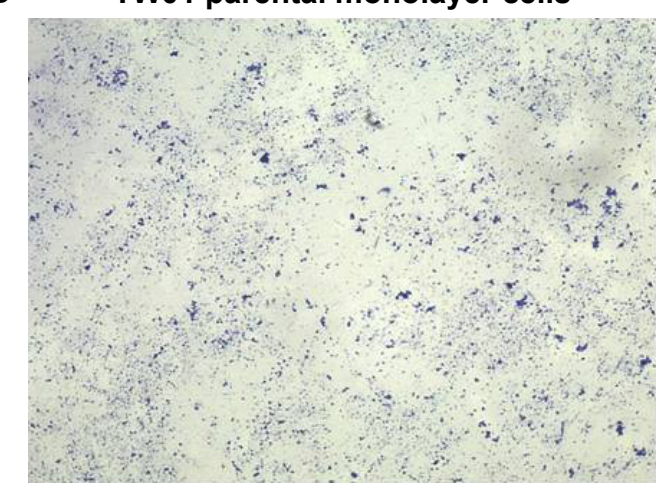

C

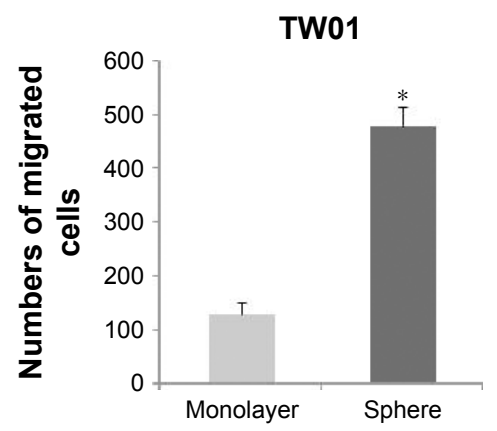

D

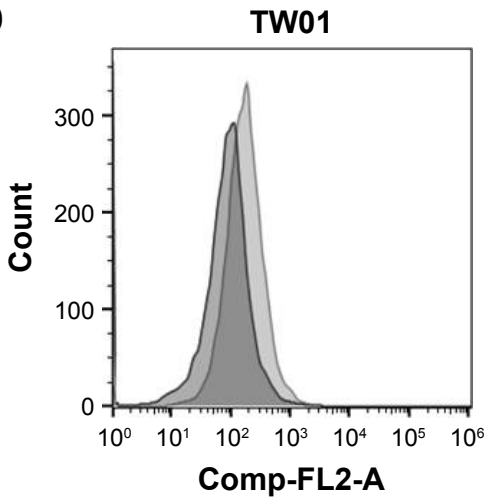

TW01 sphere forming cells

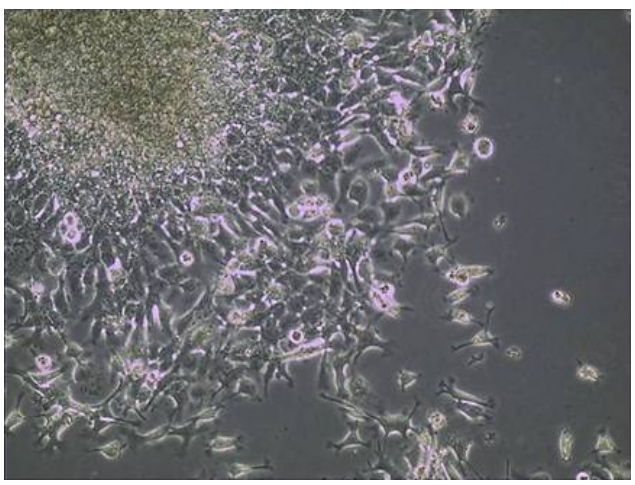

TW01 sphere forming cells

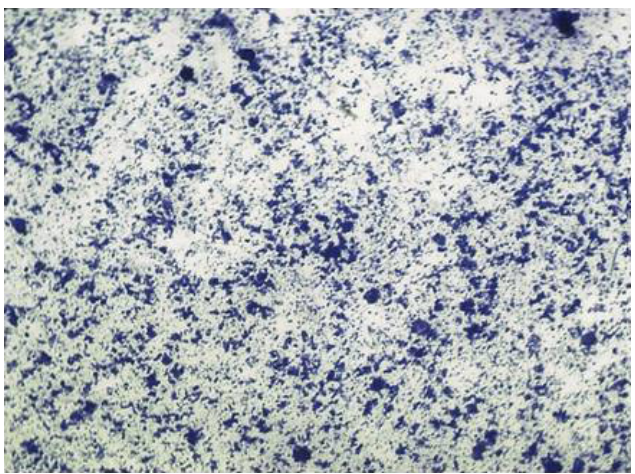

TW06
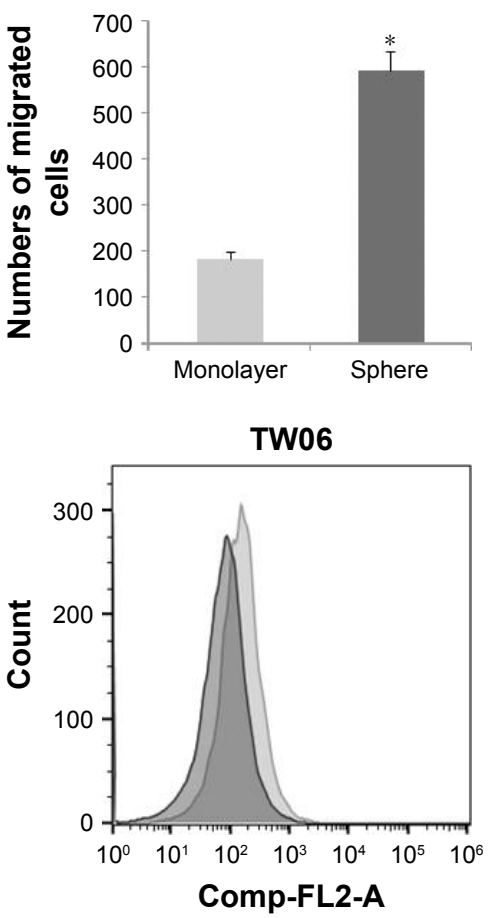

Sphere $\square$ Monolayer

Figure I Characterization of EMT of NPC sphere-forming cells. Morphology and migratory states of the TW0I and TW06 cell lines.

Notes: (A) The morphology of TWOI monolayer and sphere-forming cells reattached to $10 \mathrm{~cm}$ dishes was examined using a light microscope. (B) The ability of the TW0I monolayer and sphere-forming cells to migrate was determined using the Transwell system. (C) Migration was more prominent in the sphere-forming cells than in the monolayer cells. Replicate data from representative experiments are presented $(* P<0.05)$. (D) E-cadherin expression was detected through flow cytometry in TW0I and TW06 monolayer and sphere-forming cells.

Abbreviations: Comp, compensation; EMT, epithelial-mesenchymal transition; NPC, nasopharyngeal carcinoma. 
A

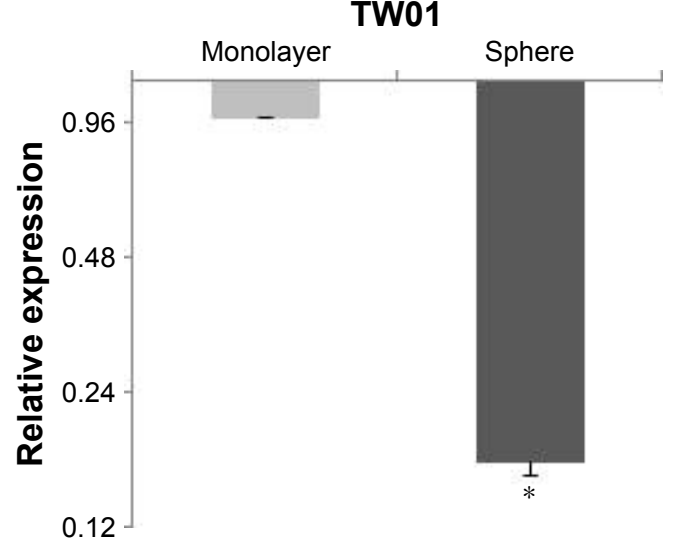

B

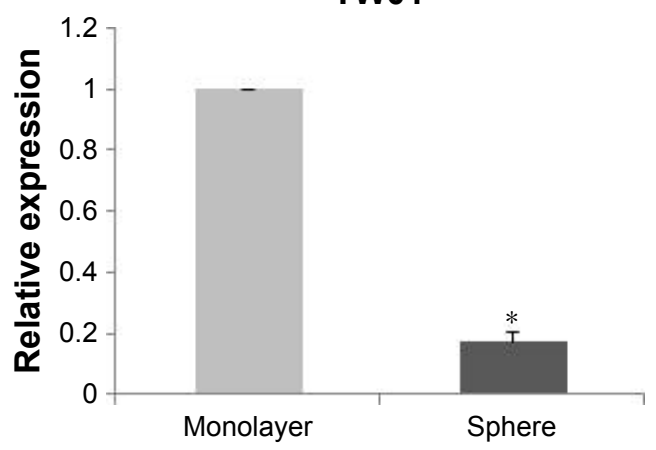

TW06

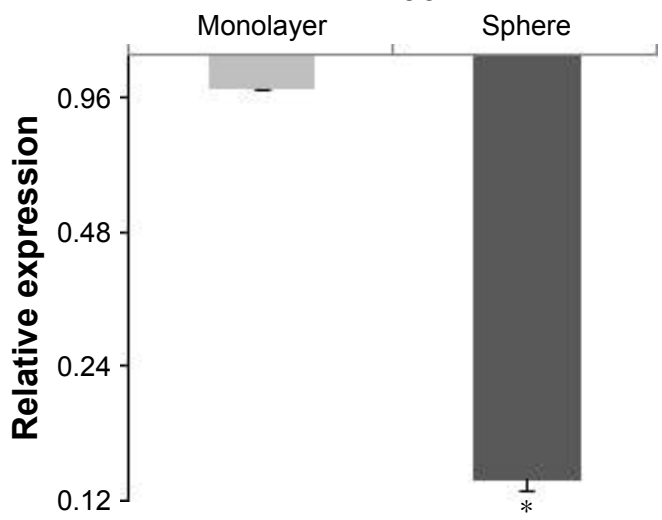

TW06

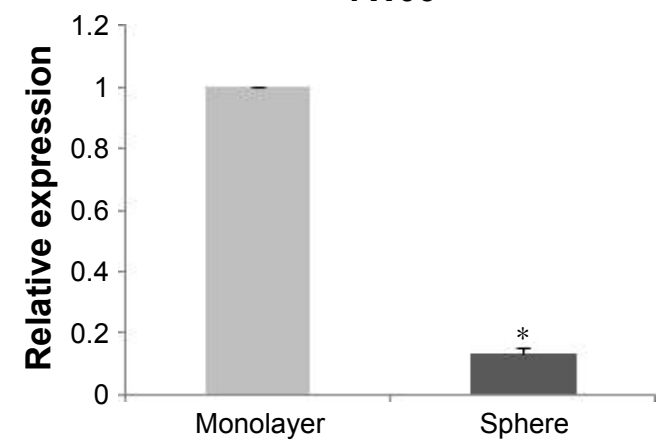

Figure 2 miRNA expression analysis performed using miRNA arrays and qPCR.

Notes: (A) The miRNA expression pattern of the NPC cells (monolayer and sphere-forming cells) was assessed using an miRNA array system. (B) The miRNA array results were confirmed through real-time PCR. The expression of miR-328 was lower in the monolayer cells than in the sphere-forming cells, which was consistent with the miRNA array results $(* P<0.05)$.

Abbreviations: miRNA, microRNA; NPC, nasopharyngeal carcinoma; qPCR, quantitative polymerase chain reaction.

aggressive behavior by reducing the expression of $\mathrm{CD} 44$ in NPC cells.

\section{CD44 is a direct target of miR-328 in NPC}

Because miR-328 inhibits NPC cell migration, EMT change, and CD44 expression, we further examined whether it exerts

A

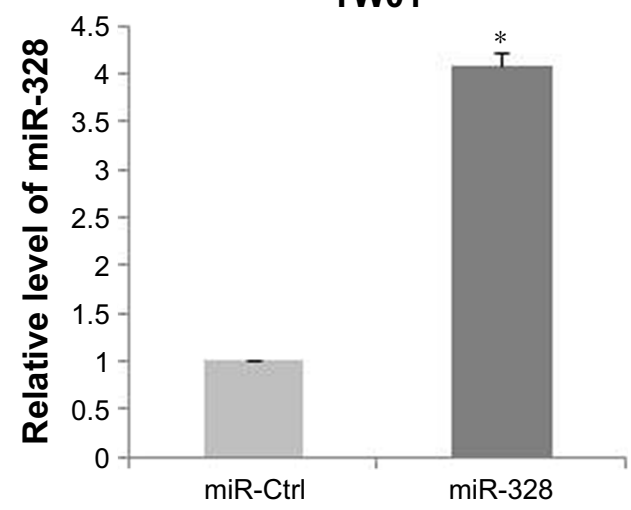

its influence on migration by targeting CD44. A luciferase assay was performed to determine whether miR-328 directly targets CD44 and affects NPC cell migration. The activity of the luciferase-expressing plasmid containing the $3^{\prime} \mathrm{UTR}$ of CD44 was significantly suppressed in the mimic group compared with that in the control group (Figure 5A). Furthermore, Western blot analysis revealed that CD44

Figure 3 (Continued)

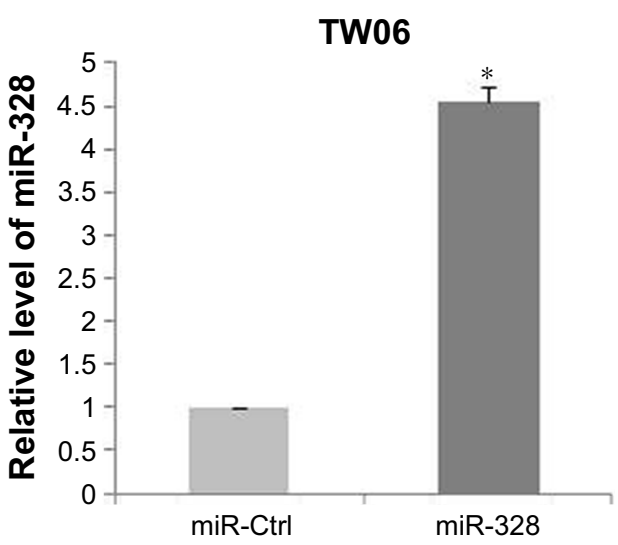


B

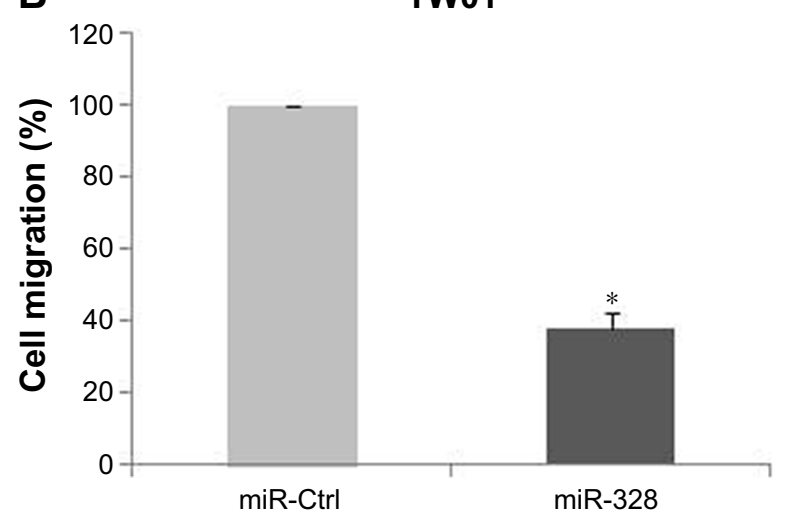

TW06

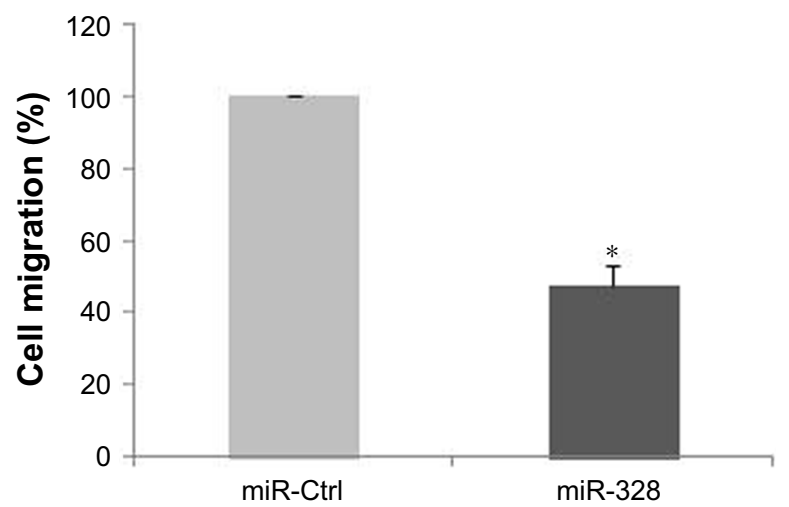

Figure 3 Overexpression of miR-328 influences the migratory ability of TW0I and TW06 sphere-forming cells.

Notes: (A) TW0I and TW06 sphere-forming cells were transfected with an miR-328 mimic to achieve miR-328 overexpression, and the levels were analyzed through real-time PCR. (B) The Transwell assay was used to evaluate migratory ability, revealing decreased migration after the overexpression of miR-328 in TW0I and TW06 cells. The data represent the average number of cells that migrated in a representative experiment. The experiments were conducted in triplicate, and the data presented are the mean values $\pm S D(* P<0.05)$.

Abbreviations: Ctrl, control; miR, microRNA; PCR, polymerase chain reaction.

protein levels were significantly decreased in the mimic group compared with the control group (Figure 5B). These results indicated that miR-328 could considerably inhibit cell migration and suppress EMT by directly targeting CD44 in NPC cells.

\section{Discussion}

Identification of specific miRNAs in tumors and their targets is crucial for understanding their roles in cancer cell growth and progression, and may be helpful in exploring novel therapeutic targets. Strategies for inhibiting the aggressiveness of several human cancers based on miRNAs have been proposed by recent studies. ${ }^{21-23}$ The roles of dysregulated miRNA in the pathogenesis of NPC have been reported; specifically, miRNAs play crucial roles in tumorigenesis, and their aberrant expression may influence NPC cell growth and metastasis. Several studies have emphasized the importance of change in the expression of miRNAs that could serve as diagnostic and therapeutic biomarkers in NPC. ${ }^{24-26}$

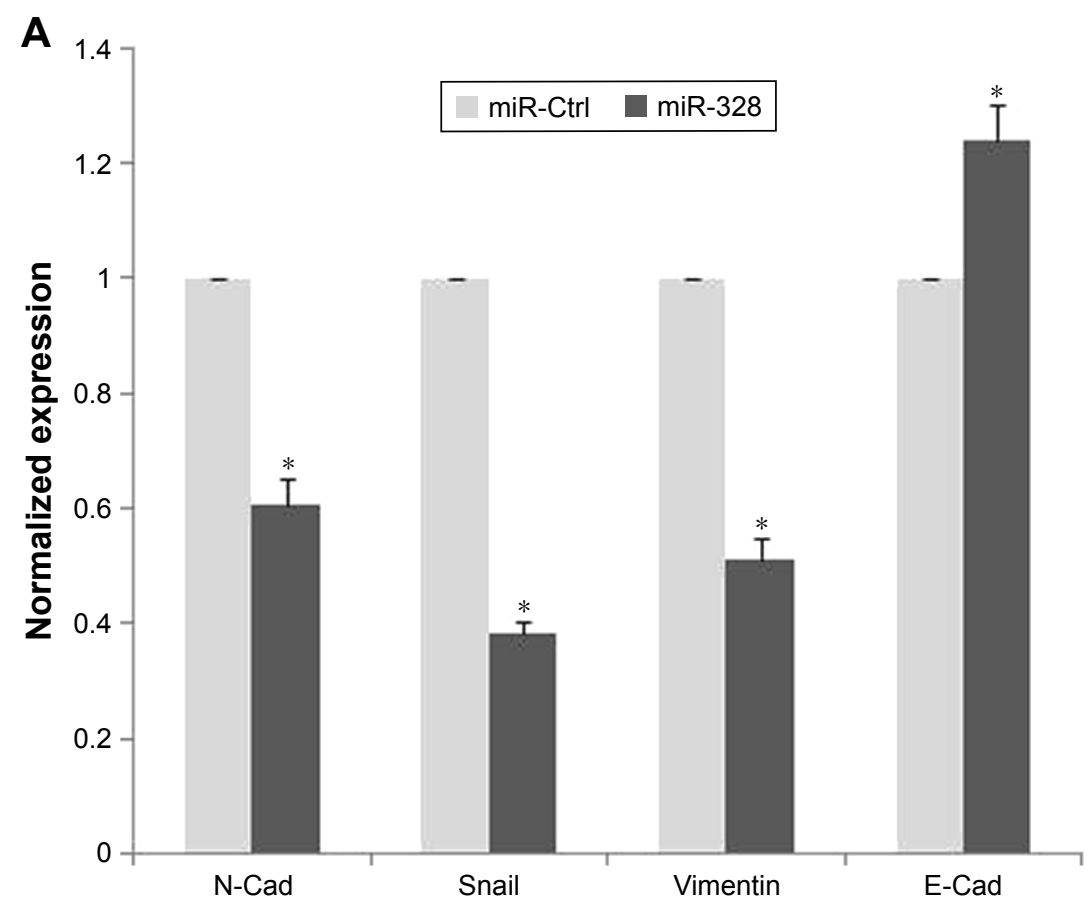

Figure 4 (Continued) 
B

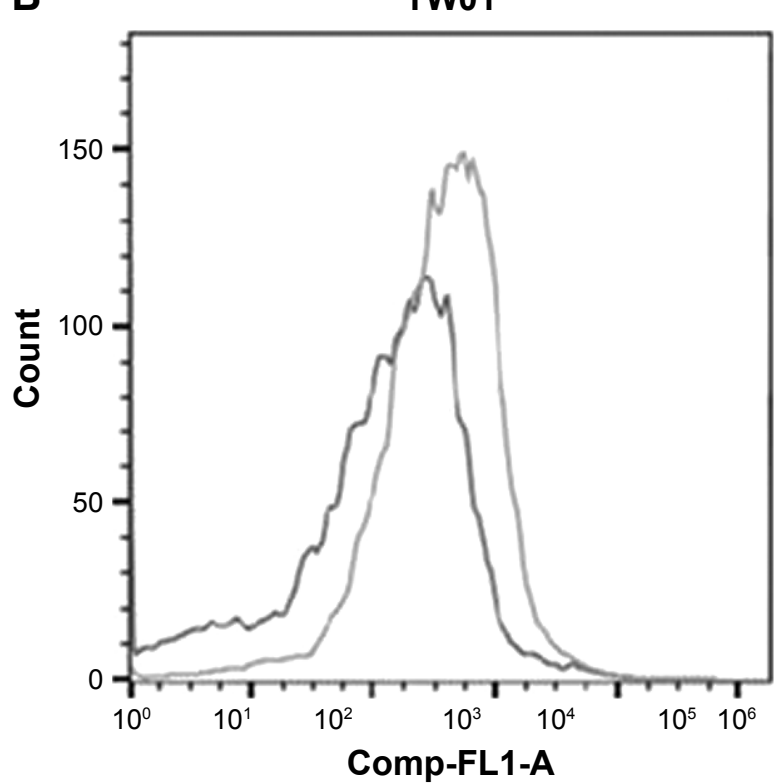

TW06

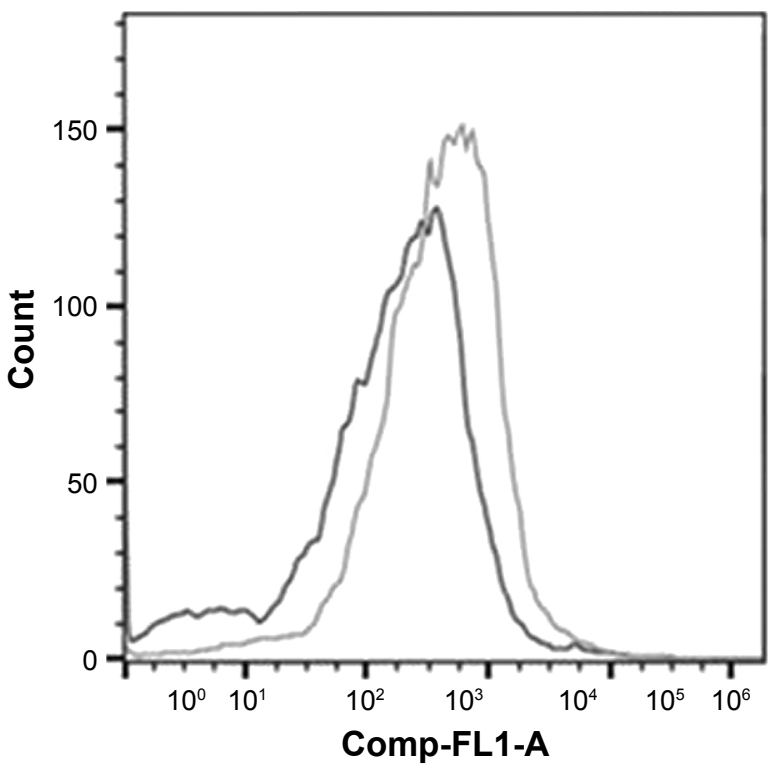

miR-328 - miR-Ctrl

Figure 4 Expression of miR-328 mediates the expression of EMT-related markers and CD44 in NPC cells.

Notes: (A) Relative mRNA expression levels of E-cadherin, N-cadherin, Snail, and vimentin in TW0I cells following transfection with the miR-328 mimic. $* P<0.05$. (B) Overexpression of miR-328 in TW0I or TW06 cells decreased the expression of CD44 as evaluated through flow cytometry.

Abbreviations: Comp, compensation; Ctrl, control; EMT, epithelial-mesenchymal transition; miR, microRNA; NPC, nasopharyngeal carcinoma.

Studies have reported miR-328 to be frequently dysregulated in several types of cancers such as lung, esophagus, cervix, and prostate cancers. ${ }^{27-30}$ However, a limited number of studies have examined the functions and mechanisms of
miR-328 involved in metastasis during the EMT process. In this study, we observed that miR-328 was downregulated in NPC sphere-forming cells that exhibited EMT characteristics, providing evidence that miR-328 expression influences the

A

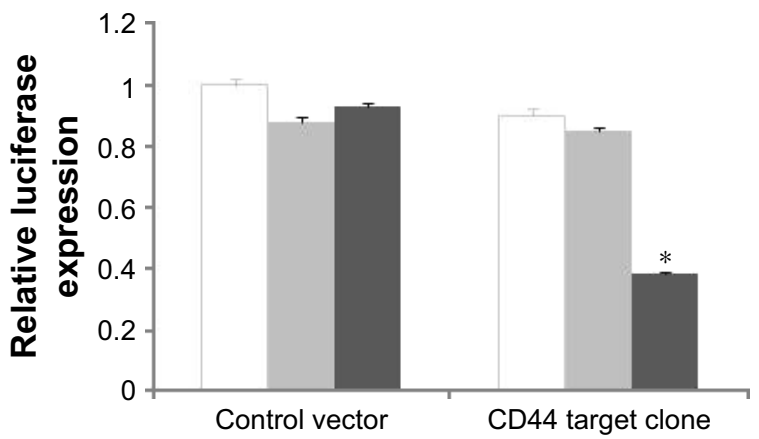

$\square$ Blank control $\square$ miR-328 mimic-NC $\quad$ miR-328 mimic

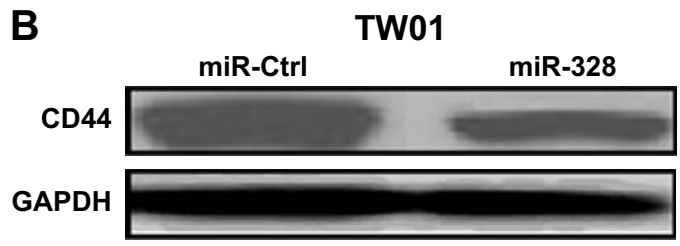

TW06

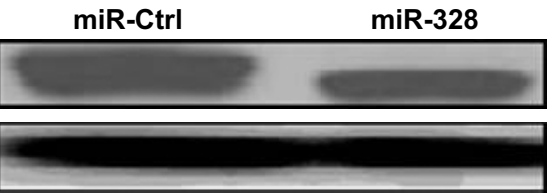

Figure 5 CD44 is involved in the regulation of cell migration by miRNA-328.

Notes: (A) Expression of a luciferase-expressing plasmid containing the $3^{\prime}$ untranslated region of CD44 was significantly suppressed in the miR-328 mimic group compared with that in the control group. ${ }^{*} P<0.05$. (B) Western blot analysis revealed that the CD44 protein level was significantly decreased in the miR-328 mimic group compared with that in the control group.

Abbreviations: Ctrl, control; GAPDH, glyceraldehyde-3-phosphate dehydrogenase; miRNA, microRNA; NC, negative control. 
migratory ability of NPC cells. Additionally, overexpression of miR-328 induced through transfection with a synthetic miR-328 mimic lowered NPC cell migration significantly. Moreover, the inhibitory effects were associated with enhanced E-cadherin levels and downregulated expression of mesenchymal markers such as N-cadherin, Snail, and vimentin. Studies have shown that miR-328 is involved in tumorigenesis and tumor progression. Yuan et al reported that overexpression of miR-328 effectively attenuated the migratory and invasive abilities of malignant cells. ${ }^{31}$ Our results are consistent with previous findings, implying that the expression of miR-328 could reverse aggressive behavior and change the expression of EMT markers in NPC cells.

The decreased CD44 expression in NPC cells transfected with the miR-328 mimic was proved through flow cytometric analysis. We performed luciferase reporter assays to clarify that CD44 was a direct and functional target of miR-328, with miR-328 binding to its 3'UTR. CD44 has been implicated as a crucial functional marker in tumor stem cells and was associated with EMT events in breast, ovarian, colon, pancreatic, and head and neck cancers. ${ }^{32-36}$ Additionally, our previous studies have indicated that the expression of CD44 may contribute to stemness, EMT, and cancer cell progression in NPC. ${ }^{37,38}$ Thus, CD44 may represent an attractive target for suppressing tumorigenesis and metastasis in numerous types of cancers. Our results also provide a clear understanding of the roles of miR-328 in migration and metastasis, suggesting that CD44 could be targeted to inhibit the migration of NPC cells and reverse EMT.

In summary, this study demonstrated that miR-328 could target CD44 in NPC cells and effectively suppress their migratory ability and the EMT phenotype. The results indicate that miR-328 plays a vital role in tumor progression and it may serve as a potential prognostic marker and therapeutic target for NPC. Further evaluation of metastatic cells from primary NPC tumors to determine the underlying mechanism of miR-328 is warranted.

\section{Acknowledgment}

This study was supported by grants from the Department of Health, Taipei City Government (104XDAA00089 and 105XDAA00086).

\section{Disclosure}

The authors report no conflicts of interest in this work.

\section{References}

1. Lung ML, Cheung AK, Ko JM, Lung HL, Cheng Y, Dai W. The interplay of host genetic factors and Epstein-Barr virus in the development of nasopharyngeal carcinoma. Chin J Cancer. 2014;33:556-568.
2. Lee AW, Fee WE Jr, Ng WT, Chan LK. Nasopharyngeal carcinoma: salvage of local recurrence. Oral Oncol. 2012;48:768-774.

3. Wang Y, Ding W, Chen C, Niu Z, Pan M, Zhang H. Meta-analysis of concurrent chemoradiotherapy in the treatment of locoregionally advanced nasopharyngeal carcinoma. J Cancer Res Ther. 2015;11(Suppl 2): C191-C195.

4. Lan M, Chen C, Huang Y, et al. Neoadjuvant chemotherapy followed by concurrent chemoradiotherapy versus concurrent chemoradiotherapy alone in nasopharyngeal carcinoma patients with cervical nodal necrosis. Sci Rep. 2017;7:42624.

5. Su Z, Mao YP, Tang J, Lan XW, OuYang PY, Xie FY. Long-term outcomes of concurrent chemoradiotherapy versus radiotherapy alone in stage ii nasopharyngeal carcinoma treated with IMRT: a retrospective study. Tumour Biol. 2016;37:4429-4438.

6. Calin GA, Croce CM. MicroRNA signatures in human cancers. Nat Rev Cancer. 2006;6:857-866.

7. To KK. MicroRNA: a prognostic biomarker and a possible druggable target for circumventing multidrug resistance in cancer chemotherapy. J Biomed Sci. 2013;20:99.

8. Chen HC, Chen GH, Chen YH, et al. MicroRNA deregulation and pathway alterations in nasopharyngeal carcinoma. Br J Cancer. 2009;100: $1002-1011$

9. Liu N, Chen NY, Cui RX, et al. Prognostic value of a microRNA signature in nasopharyngeal carcinoma: a microRNA expression analysis. Lancet. 2012;13:633-641.

10. Lee KT, Tan JK, Lam AK, Gan SY. MicroRNAs serving as potential biomarkers and therapeutic targets in nasopharyngeal carcinoma: a critical review. Crit Rev Oncol Hematol. 2016;103:1-9.

11. Wei SC, Yang J. Forcing through tumor metastasis: the interplay between tissue rigidity and epithelial-mesenchymal transition. Trends Cell Biol. 2016;26:111-120.

12. Nantajit D, Lin D, Li JJ. The network of epithelial-mesenchymal transition: potential new targets for tumor resistance. J Cancer Res Clin Oncol. 2015;141:1697-1713.

13. Pan Q, Meng L, Ye J, et al. Transcriptional repression of miR-200 family members by Nanog in colon cancer cells induces epithelialmesenchymal transition (EMT). Cancer Lett. 2017;392:26-38.

14. Zhang ZZ, Cao HC, Huang DL, et al. MicroRNA-200c plays an oncogenic role in nasopharyngeal carcinoma by targeting PTEN. Tumour Biol. 2017;39:1010428317703655.

15. Kumarswamy R, Mudduluru G, Ceppi P, et al. MicroRNA-30a inhibits epithelial-to-mesenchymal transition by targeting snail and is downregulated in non-small cell lung cancer. Int J Cancer. 2012;130: 2044-2053.

16. Ponta H, Sherman L, Herrlich PA. CD44: from adhesion molecules to signalling regulators. Nat Rev Mol Cell Biol. 2003;4:33-45.

17. Xu H, Tian Y, Yuan X, et al. The role of CD44 in epithelialmesenchymal transition and cancer development. Onco Targets Ther. 2015;8:3783-3792.

18. Lun SW, Cheung ST, Cheung PF, et al. CD44+ cancer stem-like cells in EBV-associated nasopharyngeal carcinoma. PLoS One. 2012;7:e52426.

19. Janisiewicz AM, Shin JH, Murillo-Sauca O, et al. CD44(+) cells have cancer stem cell-like properties in nasopharyngeal carcinoma. Int Forum Allergy Rhinol. 2012;2:465-470.

20. Lin CT, Chan WY, Chen W, et al. Characterization of seven newly established nasopharyngeal carcinoma cell lines. Lab Invest. 1993;68: 716-727.

21. Chakraborty C, Wen ZH, Agoramoorthy G, Lin CS. Therapeutic microRNA delivery strategies with special emphasis on cancer therapy and tumorigenesis: current trends and future challenges. Curr Drug Metab. 2016;17:469-477.

22. Vijayarathna S, Oon CE, Jothy SL, Chen Y, Kanwar JR, Sasidharan S. MicroRNA pathways: an emerging role in identification of therapeutic strategies. Curr Gene Ther. 2014;14:112-120.

23. Li C, Feng Y, Coukos G, Zhang L. Therapeutic microRNA strategies in human cancer. AAPS J. 2009;11:747-757. 
24. Wang LJ, Chou YF, Chen PR, et al. Differential miRNA expression in repeated recurrence of nasopharyngeal carcinoma. Cancer Lett. 2014;344:188-194

25. Liu N, Cui RX, Sun Y, et al. A four-miRNA signature identified from genome-wide serum miRNA profiling predicts survival in patients with nasopharyngeal carcinoma. Int J Cancer. 2014;134:1359-1368.

26. Li G, Qiu Y, Su Z, et al. Genome-wide analyses of radioresistanceassociated miRNA expression profile in nasopharyngeal carcinoma using next generation deep sequencing. PLoS One. 2013;8:e84486.

27. Ma W, Ma CN, Zhou NN, Li XD, Zhang YJ. Up- regulation of miR328-3p sensitizes non-small cell lung cancer to radiotherapy. Sci Rep. 2016;6:31651.

28. Han N, Zhao W, Zhang Z, Zheng P. MiR-328 suppresses the survival of esophageal cancer cells by targeting PLCE1. Biochem Biophys Res Commun. 2016;470:175-180.

29. Wang X, Xia Y. MicroRNA-328 inhibits cervical cancer cell proliferation and tumorigenesis by targeting TCF712. Biochem Biophys Res Commun. 2016;475:169-175.

30. Liu C, Zhang L, Huang Y, et al. MicroRNA-328 directly targets p21-activated protein kinase 6 inhibiting prostate cancer proliferation and enhancing docetaxel sensitivity. Mol Med Rep. 2015;12: 7389-7395.

31. Yuan J, Zheng Z, Zheng Y, Lu X, Xu L, Lin L. MicroRNA-328 is a favorable prognostic marker in human glioma via suppressing invasive and proliferative phenotypes of malignant cells. Int J Neurosci. 2016; 126:145-153
32. Vos MC, Hollemans E, Ezendam N, et al. MMP-14 and CD44 in Epithelial-to-Mesenchymal Transition (EMT) in ovarian cancer. J Ovarian Res. 2016;9:53.

33. Zhang Y, Wei J, Wang $\mathrm{H}$, et al. Epithelial mesenchymal transition correlates with CD24+CD44+ and CD133+ cells in pancreatic cancer. Oncol Rep. 2012;27:1599-1605.

34. Cho SH, Park YS, Kim HJ, et al. CD44 enhances the epithelialmesenchymal transition in association with colon cancer invasion. Int J Oncol. 2012;41:211-218.

35. Brown RL, Reinke LM, Damerow MS, et al. CD44 splice isoform switching in human and mouse epithelium is essential for epithelialmesenchymal transition and breast cancer progression. J Clin Invest. 2011;121:1064-1074.

36. Shigeishi H, Biddle A, Gammon L, et al. Maintenance of stem cell selfrenewal in head and neck cancers requires actions of GSK $3 \beta$ influenced by CD44 and RHAMM. Stem Cells. 2013;31:2073-2083.

37. Shen YA, Wang CY, Chuang HY, et al. CD44 and CD 24 coordinate the reprogramming of nasopharyngeal carcinoma cells towards a cancer stem cell phenotype through STAT3 activation. Oncotarget. 2016;7: 58351-58366.

38. Lin $\mathrm{CH}$, Hung PH, Chen YJ. CD44 is associated with the aggressive phenotype of nasopharyngeal carcinoma through redox regulation. Int J Mol Sci. 2013;14:13266-13281. 


\section{Supplementary material}

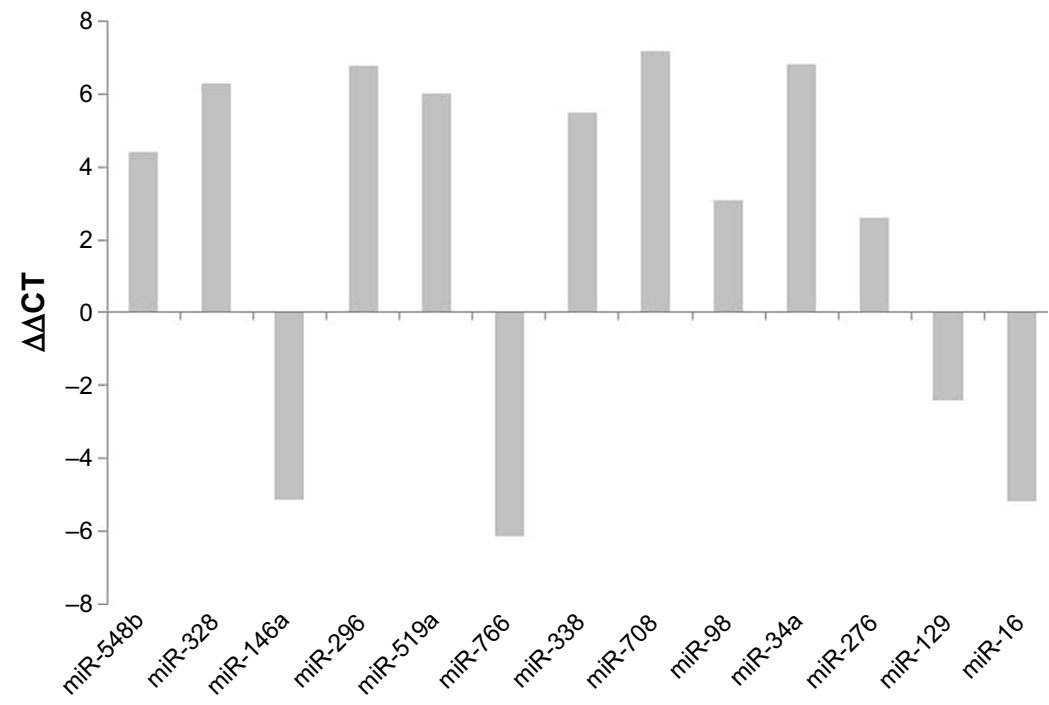

Figure SI miR expression levels of validation of TaqMan Low Density miRNA Array.

Note: The bar of each miRNA represents the results of $\triangle \Delta C T$ comparing TWOI sphere-forming cells with monolayer cells.

Abbreviations: $C T$, threshold cycle; miR, microRNA.

\section{Publish your work in this journal}

OncoTargets and Therapy is an international, peer-reviewed, open access journal focusing on the pathological basis of all cancers, potential targets for therapy and treatment protocols employed to improve the management of cancer patients. The journal also focuses on the impact of management programs and new therapeutic agents and protocols on patient perspectives such as quality of life, adherence and satisfaction. The manuscript management system is completely online and includes a very quick and fair peer-review system, which is all easy to use. Visit http://www.dovepress.com/testimonials.php to read real quotes from published authors.

\footnotetext{
Submit your manuscript here: http://www.dovepress.com/oncotargets-and-therapy-journal
} 